\title{
Cognitive Analysis of Long-Term Memory in Interpreting
}

\author{
Yingmin Guo \\ College of Foreign Languages, Xi'an Shiyou University, Xi'an, China
}

Email address:

gym@xsyu.edu.cn

\section{To cite this article:}

Yingmin Guo. Cognitive Analysis of Long-Term Memory in Interpreting. International Journal of Language and Linguistics. Vol. 4, No. 3, 2016, pp. 103-113. doi: 10.11648/j.ijl1.20160403.13

Received: April 12, 2016; Accepted: April 22, 2016; Published: May 11, 2016

\begin{abstract}
With the development of the interpretation theory, the studies on interpreting have become extremely important. In view of the importance of memory in interpretation, many scholars concentrate on short-term memory research, while ignoring the important role of long-term memory in interpretation study and practice. In this paper, the author applies the findings of cognitive psychology to probe into the strategies to improve the interpreter's long-term memory. From the perspective of cognitive psychology, interpretation is a systematic, strategic information processing process. This paper first introduces three memory systems and their characteristics, functions operations and interrelations. Then, the author analyzes the significance of long-term memory in particular, and then elaborates factors affecting long-term memory in a cognitive way. Based on this, the author proposes strategies to improve long-term memory. Finally, the author concludes that the use of these strategies can reduce the workload of restructuring, prevent mother tongue's negative transfer, reduce the pressure of language encoding and produce more accurate and fluent target language.
\end{abstract}

Keywords: Cognitive Psychology, Long-Term Memory, Interpreting

\section{Introduction}

\subsection{Purpose of the Study}

Interpretation, compared with translation, has developed fast and gained great attention from scholars and learners over the past few decades. The increasingly interdisciplinary perspective on the study of interpretation provides a new outlook for scholars in this field. As to simultaneous interpreting, it is mainly characterized by being time limited and transient. Interpreters are usually faced with dense information and high time pressure during their work. From this point of view, to transfer the information from the source language to the target language smoothly, a powerful memory capability becomes extremely significant for a qualified interpreter.

Up to now, many researchers are dedicated to the study of memory in interpretation. However, their studies mainly focus on short-term memory, and pay little heed to long-term memory. I do gain some inspirations from previous studies on memory and decide to take a bold step to apply some of the relevant theoretical findings into this field.

\subsection{The Significance of the Present Study}

Memory in interpreting generally involves three processes: recognition, retention and retrieval of information (Bao Gang, 1998: 157). In recognition process, the interpreter listens to the source language, percepts and understands the meaning, retention refers to the storage of the source language information, and retrieval of information means the preparatory work before the target language is produced. From these processes we can see that memory in interpreting is not just a simple repetition of stored information from beginning to the end. It needs an active processing and encoded message retrieval of the incoming information.

From psychological perspective, memory is an organism's ability to store, retain, recall information and experiences. Traditional studies of memory began in the fields of philosophy, including techniques of artificially enhancing memory. The late nineteenth and early twentieth century put memory within the paradigms of cognitive psychology. Since the systematic research on memory in 1960's, the relation between memory capability and interpreting remains one focal point of the study. With the increase of intercultural communications, interpretation is becoming more and more important. Many scholars and researchers have tried to find 
out effective methods for memory training from different angles. Moser-Mercer (1997), Setton (1999), Cai Xiaohong (2000) and Li Fangqin (2004) have elaborated on the role and functions of memory in interpreting. However, the research conducted by Chinese scholars is mainly about the generalization of specific memory techniques and their applications in interpretation teaching and practice over past years (Zhang Wei 2006). Moreover, the research is mainly about the relations between simultaneous interpreting and working memory, which is considered to be the focus of study and has become a fruitful field in interpreting research. In the major interpretation and translation journals in China, papers about long--term memory only accounts for a small part and the study on short-term memory often remains the focus. This is partly due to the special characteristic of longterm memory (Liu Miqing 2003). From this we see that the research on long-term memory is becoming urgent and significant.

\subsection{Research Objective and Research Questions}

In contrast to short-term memory, the role of long-term memory has received relatively less attention in interpretation practice and research. As a result, the focus of short-term memory shadows the importance of long-term memory. In order to clarify the underestimated role and function of long-term memory, this paper focuses on longterm memory and aims at setting a right position for longterm memory in interpreting. The author elaborates the significance and functions of long-term memory in interpreting and proposes strategies to improve it. As it is a relatively new field, this paper is expected to draw more attention to long-term memory and enrich the research on human memory in interpreting. This paper attempts to answer the following questions:

1. How long-term memory functions in interpreting?

2. What factors influence long-term memory in interpreting?

3. What strategies can be adopted to improve interpreter's long-term memory?

\section{Literature Review}

\subsection{Research on Cognition, Cognitive Psychology and Interpreting Abroad}

The concept of cognition is closely related to abstract concepts such as mind and intelligence, and it is used to refer to the mental functions, mental processes (thoughts) and states of intelligent entities such as humans, human organizations and highly autonomous machines. In contrast with the behavioral psychologists, cognitive psychologists studied the internal mechanisms and processes that can not be observed such as memory, storage, extraction and memory changes. Cognitive psychology is a psychological thought, branch and development of psychology rising in midtwentieth century. In the 1970's, it became one of the main psychology research in the west. Cognitive psychology is the study of human higher mental processes, primarily cognitive processes, such as attention, perception, imagery, memory, thinking and language.

As cognitive psychology covers the scientific study of mental processes, the inter-disciplinary development in linguistics, biological psychology and anthropology contribute to the research on cognitive psychology. Since interpreting is a kind of communication and information processing, and its process also covers the scope of cognitive psychology such as memory and attention. This implies that findings of cognitive psychology can also be applied to the study of interpreting. Actually, the research of interpretation has benefited from the development of cognitive psychology. Some findings and concepts in interpretation originate from cognitive science and great achievements have been made in interpretation studies. Daniel Gile's famous "effort model" can best explain the adoption of cognitive concept as his model is based on the "attention" in cognitive psychology, and Kahneman's "energy assignment pattern". In addition to the "effort model", there are other findings and theories including the interpretive theory and the informationprocessing theory and the latter still remains influential for current interpretation research. Therefore, the introduction and application of cognitive psychological studies is bound to broaden interpreting research and make more achievements.

\subsection{Research on Cognition, Cognitive Psychology and Interpreting in China}

As we discussed above, the core focus of cognitive psychology is on how people acquire, process and store information. There are numerous practical applications for cognitive research, such as ways to improve memory, how to increase decision-making accuracy, and how to structure educational curricula to enhance learning.

The research on cognition and cognitive psychology has remained a new field until 1990's in China. Since then, several influential cognitive theories have been first introduced in Chinese Translators, Chinese Science\& Technology Translators and other main articles and journals. $\mathrm{Hu}$ Gengshen stated that the development of cognition will broaden the research of interpretation in particular. In 2001, Xiao Xiaoyan introduced Gile's multiple task model and she pointed that the model was based on the concept of cognition. In the same year, Wu Yicheng also did not economize on words to evaluate the article of Cognitive Process of Interpreting, which gave us insights into the cognition development. Based on empirical study, GaoBin \&Chai Mingjiong (2009) points that the study of cognition will attract more attention in the new century. Zhong Weihe (2009) states that cognitive research achievements were mainly reflected on the field of cognitive psychology. Using a cognitive view, $\mathrm{Xu}$ Ming elaborated deverbalization in conference and simultaneous interpretation in 2010, which gave us a better understanding of cognition. Xuming pointed that cognitive process was quite complicated and interrelated with other fields such as language acquisition, neuroscience and psychology. As to the influence of cognition on other 
field research, many scholars have written articles to illustrate it. Zhang Wei (2009) pointed that cognition was a pretty important factor in interpretation. Moreover, Bao Xiaoying (2005), Liu Guiying (2006), Han Ling (2007) and Gao Fen (2007) analyzed the basis of cognitive psychology and their applications in interpreting.

\subsection{Research Findings of Interpretation and Memory Home and Abroad}

Since Cognitive psychology is the study of psychological process about people's attention, perception, memory, thought and expression, which also refers to information processing of human being. Interpreting is the use of cognitive knowledge, to analyze, synthesis, understand, and express the incoming information. We can conclude that all aspects of this process, in fact, is not a single present or a list of the order one by one, but intertwined with a complex mental process.

Gile (2000) pointed that the study of memory ability has been the focus in interpreting. As to the qualifications required for an interpreter, Phelan proposed that: "the interpreter needs a good short-term memory to retain what he or she has just heard and a good long-term memory to put the information into context. Ability to concentrate is a factor as is the ability to analyze and process what is heard."(Phelan, 2001:4-5) It is obvious that memory ability is crucial for qualified interpreters and trainees. Studies on memory in interpreting have gained attraction in western research community. In 1968, Atkinson and Shiffrin proposed information-processing model. Based on this theory, memory is divided into: sensory memory, short-term memory and long-term memory. Craik and Lockart (1972) proposed levels-of-processing approach of memory in interpreting. Watson (1999) proposed a general model of memory for simultaneous interpreting based on Baddeley's working memory theory, which was put forward in 1990. In spite of this, the study mainly focuses on the general description of memory.

Owing to the importance of memory in interpreting, many scholars in China have written articles in this field. There are five articles in CNKI database written on memory in interpreting from 2003 to 2004 ( Han Zhenyu, Han Lifang 2003; Han Xiaoming,2004; An Xinkui,2004; Li Fangqin,2004; and Ma Yingmai,Sun Changyan,2004). There are other articles and publications written on memory including Liu Wenhong, 2005, Xu Mingying, 2006, Tang Simin, 2006, Kong Jufang, 2006, XuHan, 2007, GaoFen, 2007, Yang Xianming, 2007, Yin Xiaojie, 2008, Liu Jinyu, 2009 and Zhang Fayong, 2010. Among these papers, all of which are about either general memory training or short-term memory, while ignoring the importance of long-term memory in interpreting.

\subsection{The Interrelations Between Three Memory Systems in Interpreting}

From the cognitive psychological perspective, memory is often divided into sensory, short-term and long-term memory in accordance with the duration. Sensory memory is concerned with the perception of things and its duration is very short. Studies have shown that information can only be kept from 0.25 to 2 seconds in sensory memory, it will disappear quickly and it is the shortest memory of the brain. In this stage people get information from outside. The existence of sensory memory for further processing of information is essential because sensory memory allows information to be retained to switch to the next stage of storage. As it is the first stage that people get information, it is obvious that in interpreting especially in simultaneous interpreting, the interpreters need instantaneous ability to keep information, which is the basic quality that they need to have.

Short-term memory is the process of memorization. Compared with sensory memory, its duration is a little bit longer and it is generally believed that the hold time is within seconds, the longest is just about a minute. The transient information captured by short-term memory is critical for temporary storage. Although the time for the information to be maintained is short, that period is very important. for interpreters and it is a crucial moment for interpreters to do high-intensity mental activities. At this stage, the source language is also very clear memory for interpreters and they can use this time to quickly capture a variety of instantaneous information and further relate information to the complete the change of the source language into the target one. In addition to obtaining the information that speakers are discussing, relevant information in long-term memory can be activated by short-term memory. But the short-term memory is characterized by small capacity and short duration. Therefore, the information in short-term memory should be used as soon as possible, otherwise, it will be forgotten easily.

The capacity of long-term memory is infinite, as long as information is reviewed, it can be maintained in long-term memory. Recent studies have shown that information in longterm memory is encoded by a variety of complex state of a certain organization to be stored together. Long-term memory is like a huge library, which holds various facts, information and knowledge and its capacity is almost unlimited. In the long-term memory, the information may be saved forever. In spite of its importance in information processing, the role of long-term memory has been neglected by psychologists since German psychologist Ebbinghaus first studied memory systematically in the 19th century. Because after 1960's the study of short-term memory has been attracting extensive attention.

When the processing of linguistic information get into long-term memory stage, information on its capacity is much greater. As the information in long-term memory is empirical and things that people have heard, seen and experienced, long-term memory has a great impact on our interpretation. The more the brain store the knowledge, experience and knowledge, the better the interpreter will play in the interpretation level. 
Generally speaking, the perception of sensory memory is often related to the source language; short-term memory and long-term memory have a certain duration, so they are connected with interpreter's storing of relevant source information. With the longest duration, long-term memory has accumulating and overlapping characteristics, so it is closely related to interpreter's language learning which can be understood as linguistic knowledge and the accumulation of knowledge including extra-linguistic knowledge. As to memory, the "sensory memory" is the premise of interpretation, "short-term memory" is the key to interpretation, "long-term memory" is the basis for interpretation. (Liu Jinyu 2009).

\section{Long-Term Memory: A Facilitator to Interpreting}

\subsection{A Cognitive Study of Long-Term Memory in Interpreting}

Cognitive scientists usually study memory in a psychological way, but they tend to focus more on cognitive processes of memory and the interrelationship between cognition and memory. Long-term memory occurs when we have created neural pathways for storing ideas and information which can then be recalled weeks, months, or even years later. To create these pathways, we must make a deliberate attempt to encode the information in the way we intend to recall it later. Long-term memory is a learning process and it is essentially an important part of the interpreter's acquisition of knowledge because information stored in it may last for minutes to weeks, months, or even an entire life.

\subsection{The Significance of Long-Term Memory in Interpreting}

Long-term memory is a real data base, which is characterized by large information capacity and long duration. By retelling or elaborate information processing, the information in short-term memory enters long-term memory. Whether the information can be recalled or retrieved from long-term memory is decided by the amount of knowledge that a person has stored.

Bao Gang (2005) points that interpreting consists of three stages of memory process: recognition/comprehension, storing and information claim. For each stage, it is participated by long-term memory. In recognition and comprehension stage, long-term memory furnishes the interpreter with necessary knowledge of phonology and grammar so as to identify the specific words and sentences. Besides, long-term memory provides ample understanding for the topic of the given speech, directing the interpreter's attention to the new and key information and ruling out the distracting input. The more experiences and knowledge an interpreter has, the more successful interpretation can be. In the stage of production, the role of long-term memory also can not be neglected. Since the production in interpreting needs a reversion between two different languages, interpreters' obtained knowledge can serve to accelerate this production process.

Tarpy (1978) states that the information processing approach offers a way of dividing the memory system into sensory memory, short-term memory and long-term memory. Xiao XiaoYan (2002) points that this approach remains quite influential for scholars to study interpreting. The focus of this relatively new approach is the information transfer between source language and target language. The process involves the transfer of receiving, storing, processing and transmitting information and each part in the whole system is interrelated and interactive. From modern cognitive psychological perspective, human beings are compared to computers in the information processing. Human beings, are regarded as an active information processor and they can perceive, encode, store and retrieve information like machines during the whole processing phase. In the first phase, interpreters receive and try to comprehend incoming information. When entering the second phase, they analyze and encode information in target language. Then the next expressing phase including evaluation process will have an effect on the second analysis and encoding process. It is clear that this information processing model provides us with clear basic steps in interpreting.

\section{Factors Affecting Long-Term Memory in Interpreting}

\subsection{Attention and Long-Term Memory}

When we read through a section of a passage in a book, the highlighted section stands out, causing us to focus our interest on that part. Similarly we can listen to what someone is saying carefully while ignoring other conversations or listen to a mobile phone conversation while driving a car. How can we manage to experience all of these sensations and still focus on just one element of our environment. Cognitive psychologists hold that it is attention that allows us to "tune out" information, perceptions and sensations that are not relevant at the moment and instead focus our energy on the information which is important.

Attention is a concept studied in cognitive psychology that refers to how we actively process specific information present in our environment. Attention is the cognitive process of selectively concentrating on one aspect of the environment while ignoring other things. According to psychologist and philosopher William James, attention is " the taking possession of the mind, in clear and vivid form, of one out of what may seem several simultaneously possible objects or trains of thoughts...It implies withdrawal from some things in order to deal effectively with others" It has also been referred to as the allocation of processing resources. Attention is one of the most intensely studied topics within psychology and cognitive neuroscience.

Attention is one of the major components of memory. In 
order to move information from short-term memory into long-term memory, we need to actively attend to this information. Attention is generally accepted as the cognitive psychology concept of mental concentration effort or on mental events or sensory. William James defined it as "everyone knows what attention is. It is the taking possession of the mind, in clear and vivid form, of one out of what seem several simultaneously possible objects or trains of thought. Focalization, concentration and consciousness are of its essence (W. James, 1980,. 403-404)". He also divided attention into active attention and passive attention. According to him, the interpretation is "passive attention" and it is the bottom-up processing. Later, Broadbent further developed the theory of attention into a filter model which means that information processing is restricted by a channel capacity. To be more specific, before going into a higher processing, the coming information passes through a filter. The incoming information will be checked by filter according to its physical characteristics. Because the capacity of sensory memory is quite limited and we can not deal with all sensory cues at one time and this makes a higher processing possible. This selectivity of information is what we later called Broadbent's Bottleneck Effect.

Broadbent's Bottleneck Theory was rather popular when it was first proposed. On the basis of this theory, the selective filter and the limited channel are indispensable parts for the focalization of attention and information processing. Before the stimulus does not attend and pass through a selective filter much less enter a limited capacity channel geared to short-term memory and long-term memory, a higher stage of information processing will not happen. Later, Broadbent modified this and he gave a suggestion that the selective filter should only be confined to one of the channels for each time. Only in this way, can overloading of the message in the limited capacity channel be avoided. Among the relevant research findings, the multi-store model proposed by Atkinson\&Shiffrin remains influential as well. The model refers to sensory memory, short-term memory and long-term memory as we quoted above. The whole process of multistore model will be explained by the following chart.

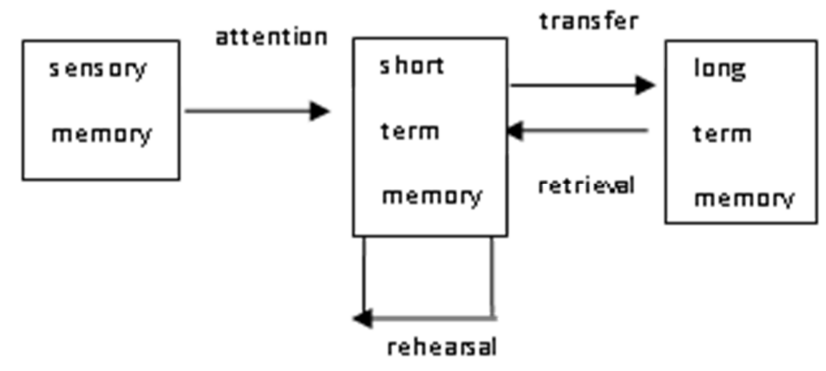

Figure 1. Multi-store Model.

\subsection{Comprehension and Long-Term Memory}

According to cognitive psychology, the interpreter's comprehension of source language is actually an information process. It means that the interpreter focus attention on the source language and process the information in order to store the processed information for later work. Bao Gang (2005) states that "Comprehension in interpretation includes analysis and synthesis; it may develop sub-consciously or consciously, and it also combines linguistic factors and nonlinguistic factors."

Memorizing process which is closely related to comprehension in interpreting is very important. Interpreting is generally accepted as recognition, retention and retrieval of information, the three types of memories work and interact together. Recognition is generally accomplished consciously by means of filtering and after the filtering some information will enter next memory storage of retention. What will be retaining information in this phase is generally affected by the interpreter's familarity with the processing information and the vividness of the source language materials. However, the process of retention is usually accompanied by forgetting as a quite common problem. The process of information retrieval can be finished by recognizing and recalling. In this phase schema plays a crucial role such as conducting the information reconstruction and directing information retrieval. To be more specific, the existing knowledge and experience in interpreter's long-term memory facilitate the comprehension. Therefore, comprehension and long-term memory are inseparable in interpreting. In a word, comprehension in interpreting is not just to understand the superficial linguistic information and not a process of coding, decoding and output, either. Therefore, we can comprehend information as long as the new information matches the background knowledge stored in our long-term memory. From this we can conclude that comprehension is not just a passive process of receiving vocal information, but an active process of reconstruction by utilizing schema while the activation of schema depends on the necessary linguistic decoding. This is the listening comprehension process in the cognitive theory. Of course the actual cognitive process is much more complex.

\subsection{Forgetting, Retrieval Failure, Fading and Long-Term Memory}

\section{Forgetting}

From forgetting where we left our keys to forgetting to answer a phone call, memory failures are an almost daily occurrence. Forgetting is so common that we typically rely on numerous methods to help us remember important information, such as jotting down notes in a daily planner or scheduling important events in our calendar.

As we are frantically searching for our missing keys, it seems that the information about where we left them is permanently gone from our memory. However, forgetting is generally not about actually losing or erasing this information from our long-term memory. Forgetting typically involves a failure in memory retrieval. While the information is somewhere in our long-term memory, we are not able to actually retrieve and remember it.

Interpterers also can not avoid forgetting no matter how hard they try to do. There are various reasons accounting for forgetting information including fading, interference, 
retrieval failure and storage problems. The most frequent reason is the difficulties in transferring information from short-term memory to long-term memory. What's more, we can forget because we are unable to recall information that stored in long-term memory

\section{Retrieval failure}

What does retrieval exactly mean? Generally, it is a process of accessing stored memories. Suppose when we are taking an exam, we need to be able to retrieve learned information from our memory in order to answer the test questions. In interpreting, the interpreter also needs to retrieve stored information to accomplish the task of recognition and recalling. There are four basic ways in which information can be pulled from long-term memory. The type of retrieval cues that are available can have an impact on how information is retrieved. A retrieval cue is a clue or prompt that is used to trigger the retrieval of long-term memory.

Retrieval theory reveals that forgetting occurs if the retrieval channels to memories are no longer available, but not because it is the true loss of memory from storage. Psychologists have found that in the encoding phase the method of people's organizing material would affect the way the material stored and the kind of working cues when people want to retrieve the material. A certain working retrieval cue makes it possible to recall the stored information from longterm memory. People's organizing and storage of materials are largely decided by how we are likely to retrieve them. So it is agreed that it is much more difficult to recall information than to recognize it. If the interpreter accomplishes recalling, he needs to reproduce early acquired information. While recognition just requires the interpreter to make sure that a previously acquired message is sensed. It is evident that both recall and recognition are crucial factors for an interpreter to trace the logical links of the speaker. This indicates that interpreters must pay attention to logical links of incoming information to avoid retrieval failure in interpreting.

\section{Fading}

Fading happens when people do not recall information any longer from their memory because of the disuse. In shortterm memory, fading can occur very quickly, even just a few seconds after some cases. When the message fades from short-term memory, it disappears because the short-term memory requires space of other incoming information. We can transfer information to long-term memory or by constantly rehearse it and focus attention on the information continually to prevent this kind of fading.

It is believed that once information has been transferred to long-term memory, it is permanently stored there. According to cognitive psychology, the information that really fades from long-term memory is the link. That means it is not the nonexistence of the information but just we can not find it and find a way to retrieve it. From this we can conclude that we should encode the information as meaningfully as possible, retrieve it frequently, restore it actively whenever we do retrieve it, and use effective memory search strategies to prevent this kind of fading.

\subsection{Mother Tongue Interference and Long-Term Memory}

Many theorists believe that the reason for forgetting is not the lost of memory from storage but just other message gets in the way of the information that people want to remember. This is interference and it occurs when information gets confused with other information in our long-term memory. Proactive interference and retroactive interference are the two most common types.

When the earlier obtained information disrupts the recall of the later obtained information, proactive interference happens. Proactive interference occurs because current information is mixed up with previously learned, similar information. On the contrary, when the retrieval of obtained earlier message is disrupted by the later acquired message, retroactive interference occurs. Retroactive interference happens because previously learned information is mixed up with new and somewhat similar current information. In interpreting practice, proactive interference means that the middle part of the material can be affected by the beginning part of the information, therefore, the middle part of the source information may be forgotten and interpreters can have a better performance on the recalling of beginning part of the source information. Similarly, retroactive interference means that the information of last may be retrieved from long-term memory and much attention should be paid on the middle part of the source information in particular.

The differences between native and target languages would lead to interference as well, also known as negative transfer. We noticed learner's use of prior linguistic information (chiefly his mother tongue) or some physically carryover of native language surface to a second language context. For example: "to touch the society", "grasp time firmly."( direct transfer from Chinese collocation to English collocation) Since English and Chinese have many differences in that English belongs to the Indo-European, and Chinese belongs to Sino-Tibetan language family. The characteristic of English syntax is hypotaxis while Chinese is parataxis and there was no subject or a subject is often hidden in Chinese. This makes no one is immune to the interference of mother tongue.

Since the task of interpretation is to use the target language to express the content of the source language, namely: to express the meaning expressed in the source language with a completely different vocabulary and sentence patterns. While listening to the speech of others, interpreters must forget source language term immediately and analyze the full meaning of the source language. Therefore, interpreters' memory of source language plays an important role in interpreting. Because information in long-term memory can be kept long and the knowledge in it have a crucial impact on interpretation. It is evident that the interference of mother tongue in long-term memory is inevitable in interpreting.

\subsection{Extra-Linguistic Factors and Long-Term Memory}

According to cognitive psychology, the interpreting process is really the interaction process of the three kinds of 
memory systems. Sensory memory is the premise of interpretation, short-term memory is the key to interpretation and long-term memory is the basis for interpretation as we stated earlier. In spite of the importance of short-term memory in interpreting, long-term memory plays an indispensable role to short-term memory. As long-term memory is mainly made up of our experience and knowledge of the world. It is much easier for interpreters to comprehend source language input with relevant background stored in long-term memory. In addition, this will reduce the stress laid on short-term memory and interpreter's pressure.

As we discussed, interpretation is not just the equivalence from source language to target language. The whole process involves the recognition, recalling and reproduction of information and long-term memory plays an important role in retrieving information. In order to accomplish interpretation tasks, interpreters need to have both linguistic knowledge and extra-linguistic knowledge. Researchers have paid more attention on non-linguistic factors on the study of the interpreting especially simultaneous interpreting over past years. Moser (1978) established a mode of semantic information processing flow chart to explain the process of interpretation, emphasizing the role of long-term memory. Miller (1956), Shacter (1987), Baddeley (1990) have done detailed studies on short-term memory and long-term memory. Shacter (1987) once divided long-term memory into two parts: explicit memory and implied memory. Explicit memory is divided into semantic memory and episodic memory. We know that long-term memory can store information more stable and permanently. In interpreting, mainly simultaneous interpreting, the interpreter mainly relies on semantic memory in which encyclopedia knowledge is stored.

In Daniel Gile's famous $\mathrm{C}=\mathrm{KL}+\mathrm{ELK}$, he explained that comprehension of incoming information is based on linguistic and extra-linguistic knowledge. In the equation, C, KL and ELK stand for comprehension, linguistic knowledge and extra-linguistic knowledge respectively. In a word, correct comprehension of source language input is partly due to extensive extra-linguistic knowledge. Because it is mainly stored in long-term memory, thus extra-linguistic knowledge will affect long-term memory performance and also has a indirect influence on short-term memory performance.

\section{Strategies to Improve Long-Term Memory in Interpreting}

\subsection{Activation of Knowledge}

Long-term memory serves as a database from which we acquire new knowledge. Once information has been encoded and stored in long-term memory, it must be retrieved in order to be used. The knowledge we store in long-term memory has great impact on our perceptions of the world, and affects information we are exposed to. Long-term memory differs from short-term memory because information can be stored for longer period of time. Therefore, the interpreter need to have a fast response to retrieve the incoming information stored in long-term memory because interpreting is characterized by high time pressure and there is no aid to resort to. Researchers have proved that people can only recognize and recall familiar materials in interpreting. Thus, it is pretty important for interpreters to activate knowledge frequently.

The other reason for interpreters to activate knowledge is that most of the information in a person's long-term memory is passive. In order to make as much as information easy and efficient to retrieve in interpreting, interpreters need to activate their passive memory frequently.

When conducting activation, we should pay attention to mainly three aspects: activation of both languages, activation of the field knowledge and that of encyclopedia knowledge.

Activation of language knowledge requires interpreters keep reading and listening as much as possible in both languages so that they can respond quickly when coming up with new expressions, idioms and quotations that are not frequently used

Activation of field knowledge means that interpreters should try to get familiar with certain field knowledge, especially the specific terminology before an interpretation task. They can use resources from Internet, library or others. Thus, when reformulating information, their long-term memory can retrieve efficiently what they have stored beforehand.

As to activation of encyclopedia knowledge, interpreters should cover as much as they can in different areas and keep their knowledge updated so that their long-term memory has a larger and larger database to rely on during interpreting.

\subsection{Prefabricate Chunk Accumulation}

Corpus-based study revealed that $90 \%$ of the natural discourse is made up by those semi-fixed structures, which are between words and phrases. The studies also have found that training language learner's "chunk" awareness is an important method to improve interpretation ability. Becker (1975) once said that we express our ideas from brain out of those prefabricated phrases and after a minor processing they can be combined into sentences. Then, this becomes influent languages to fill some space of our concept. It means that the unit of language storage and output is not the single word but those fixed and semi-fixed mode of "chunk". Lewis (1993) pointed that large number of languages consist of "chunks", these chunks will combine to produce a continuous coherent text, and only a small part of the spoken sentence is a new creation without using chunks. The chunks are usually divided into the following types:

1. Collocation or Polywords for example: 经济全球化, 可持续发展，天人合一 by comparison, nevertheless, in one word, on the contrary etc.

2. Institutionalized Expressions: mainly includes proverbs, aphorisms, communication sets of words and idioms

From the above categories, we can see that the chunk is like prefabricated chunk of languages. It is ideal for storage and output, which is semi-fixed matchers. For example: “建 
设有中国特色的社会主义”: to build socialism with Chinese characteristics，“具有中国特色的社会主义” has frequent occurrence in newspapers and articles. It has a relatively stable grammatical structure and the meaning also has a specific range. It will be used as a conventional block eventually. As interpreters often come across chunks in interpretation, mastery and application of these chunks are naturally of vital importance for interpreters to develop and enhance their memory ability.

As we analyzed above, long-term memory provides the framework to which we attach new knowledge. It is different from short-term memory in that information can be stored for longer period of time. During the process of interpreting, if the interpreter is familiar with incoming information, his long-term memory will be activated and less difficulties will exist in interpreting. That is to say, accumulation of prefabricate chunks has relation and aid for long-term memory. It is advisable for interpreters to do this life-long accumulation as long as they do interpreting even for a day. The accumulation includes proverbs, poetry and institutionalized expressions like: "on behalf of...I bid a warm welcome to you all gathered here to participate...," "I wish... a complete success," "I wish... a pleasant stay," "with its ancient civilization of China enjoys a long tradition of brilliant civilization etc." All of these are considered prefabricated chunks set in the context of language, and these are worth the attention for all the interpreters. We can conclude that the application of these chunks will shorten the process of language restructuring and target language will become more accurate and fluent in interpreting. Therefore, accumulating large number of chunks is an effective method to improve interpreters' long-term memory.

After a long period interpretation practice, the author found that with the training of prefabricated chunks accumulation, the memory is much better. We also found that this kind of training can greatly increase the interpreters' reaction rate, thereby make interpreters more confident. The best interval for prefabricated chunks set training is three to five minutes and once again the first memory with the time interval should not exceed two hours. This process, involves "semantic memory" of long-term memory of interpreters.

Another advantage of this kind of chunk accumulation is that the prefabricated chunk in the interpreter's memory will form an "automated" process such as "trust and love", "ensure social equity and justice through development", "strive for scientific development, which puts people first and pursue comprehensive, balanced and sustainable development" etc. This automated response will make the efficiency of code switching rate increase greatly. Therefore, in the training process, mastery of memory theory knowledge combined with accumulation of prefabricate chunks become quite important for interpreters to improve their long-term memory.

\subsection{The Use of Mnemonics Systems}

Mnemonics systems are clues of any kind that help us remember something, usually by helping us associate the information we want to remember with a visual image, a sentence, or a word. More specifically, it is simply a way to remember information. For example, we might associate a term we need to remember with a common item that we are very familiar with. The best mnemonics are those that utilize positive imagery, humor or novelty. We might come up with a rhyme, song or joke to help remember a specific segment of information. In medieval times they were considered as the magic arts. Today, they are used as a memory improvement way. The basic principle of mnemonics is to use as many of the best functions of our brain as possible to store information. Our brains evolved to code and interpret complex stimuli such as images, colors, structures, sounds, smells, tastes, touch, positions, emotions and languages. We use these to make sophisticated models of the world we live in. A very simple example is the " 30 days hath September" There are several mnemonics techniques such as acronym, association, visual image and chunking, etc. We just introduce a common way of chunking in interpreting here.

\section{Chunking}

The process of transferring information from short-term memory to long-term memory involves the encoding or consolidation of information. This is not a function of time, that is, the longer a memory stayed in short-term memory, the more likely it was to be placed into long-term memory; but on organizing complex information in short-term memory before it can be encoded into long-term memory. In this process of organization, the meaningfulness or emotional content of an item plays a greater role in its retention into long-term memory. As instructional designers, we must find ways to make learning relevant and meaningful enough for the learner to make the important transfer of information to long-term memory.

On a more concrete level, the use of chunking has been proven to be a significant aid for enhancing the short-term memory transfer to long-term memory. As short-term memory's capacity is limited to about seven items, regardless of the complexity of those items we can break a long list of numbers or other types of information into smaller, more manageable chunks. It allows the brain to automatically group certain items together, hence the ability to remember and learn become better.

\subsection{Schema Construction}

Schema is a cognitive term that means the mental models of the world. It is used to refer to the organization and information structure in people's memory. Cook (1992) defines schema as one's prior experience in his or her memory that influences how incoming information is processed and remembered. From the definition we can conclude that schema is closely related to people's long-term memory. Since schema is the representation of people's world knowledge and information in long-term memory is stored in interrelated networks of schemas. These, in turn, form intricate knowledge structures. Related schemas are linked together, and information that activates one schema also activates others that are closely linked. This is how we 
recall relevant knowledge when similar information is presented. These schemas guide us by diverting our attention to relevant information and allow us to disregard what is not important. Since Long-term memory storage is organized into schemas, we should activate existing schemas before presenting new information. Long-term memory also has a strong influence on perception through top-down processing as our prior knowledge affects how we perceive sensory information. Our expectations regarding a particular sensory experience influence how we interpret it. Thus, in the process of interpreting, schema plays an important role in the comprehension and predication of incoming information and the function of long-term memory can be explained by schema theory. Therefore, the improvement of long-term memory can be achieved by schema construction step by step. We can follow some interpretation courses here. Before the class, the topic and assignments of next class are usually given by the teacher. Trainees are required to use libraries and surf the internet to obtain information that is related to the topic. Through this work, the trainees will have large amount of resources which are relevant to the subject. After this, the class is often conducted in the form of mock conference. One person gives a speech and the other one gives a consecutive interpretation while taking notes. This can also be accomplished by simultaneous interpretation. The next period will be followed by the classmates' and the teacher's evaluation. The trainees will have more incentive to give a better performance next time if they get encouragement. After class, they are encouraged to have a encyclopedia knowledge and this accumulation process means the consolidation of schema construction which in turn will improve long-term memory naturally.

From previous discussions, we know that received information in short-term memory is likely to go into a more permanent long-term memory. However, not all of the shortterm memory can enter long-term memory and this is called the bottleneck effect as we analyzed earlier. After a certain length of time remaining, some of the information held in short-term memory will turn into the long-term memory while others are quickly lost, long-term memory of shortterm information transfer to the memory seems to be selectively accepted. This phenomenon shows that schema construction is of vital importance for interpreters to improve long-term memory.

\subsection{Cognitive Ability Cultivation}

The psychologist Piaget once proposed two different organizational functions. One is mental function which is to determine how human interact with the environment and to learn from the environment The other is cognitive structures commonly known as schema. Schema is the generalized knowledge about event, situation and objects. The schema theory is accepted as a theoretical model to explain the functions of people's long-term memory in the process of comprehension. Piaget holds that central part of learning is the psychological function including assimilation and adaptation.
People learned useful things and assimilated them into the existing cognitive schema from the environment during the process of their development. They also constantly change their behavior to adapt to the environment. Piaget believes that language ability development is after cognitive ability development. This shows that our language learning is restricted by cognitive ability and not all the external stimuli can cause reaction to go into schema. Therefore, people acquire memories about past experience through learning. Memory can become part of schema and the development of schema makes our cognitive ability develop further. As our ability to receive information is largely decided by our cognitive ability and therefore long-term memory has a direct even decisive effect on short-term memory performance. Moreover, this development process is generally dynamic but there is a relatively stable stage. People obtain information by the continuous and circular interaction with environment, and people encode and store information into long-term memory through this interaction. The development of each phase is more comprehensive than previous one and can assimilate more experience. Therefore, some information can not be left in the short-term memory and go into long-term memory, and this is mainly because the receiver's cognitive ability has not developed to a certain degree. It is obvious that we need to improve cognitive abilities.

In addition to cognitive abilities, there are other factors affecting long-term memory development, thus affect the ability of short-term memory. It is proved by experiments that more exercises can extend memory retaining time (Hebb, 1961). Short-term memory may convert to long-term memory by repetition, rehearsal, recognition, and recalling.

In summary, cognitive ability, the existing cognitive structure will affect the long-term memory strength and its retaining time, which also affects the short-term memory effects and short-term memory capacity development.

\section{Conclusion}

Interpreting usually involves recognition, retaining and reproduction of the information and long-term memory plays a significant role during the whole process.

The information in sensory memory and short-term memory includes a temporary recognition and retaining process. When the incoming new information matches or is similar to the information stored in long-term memory, the new information will be recognized and enter long-term memory. Otherwise, the information in long-term memory can not be activated.

Since long-term memory involves the whole process in interpreting and there are many factors such as attention, comprehension, forgetting and extra-linguistic knowledge affecting long-term memory. Interpreters can improve their long-term memory to have a better interpretation performance.

On the above analysis, some strategies are proposed to improve long-term memory. Frequent activation of knowledge can make memory more active. Fabricate chunk 
accumulation makes it possible to internalize knowledge for interpreters. The use of mnemonics systems of chunking can release the burden of short-term memory. Schema construction and cognitive ability cultivation will affect people's understanding of the world and interpreter's attention and memory can be guided by it. All of these strategies can improve the transient accumulation of memory effect, expand short-term memory span and enrich the contents of long-term memory so that the memory improvement will lay a solid foundation for interpretation practice.

Cognitive psychological research on memory provided a strong theoretical basis for interpretation teaching and practice. As the studies on long-term memory in interpreting have attracted less attention and we think the paper from cognitive perspective is tentative. There must be some deficiencies and limitations in this paper due to our academic strength. Since the strategies proposed to improve long-term memory in this paper were not proved in interpretation practice. Therefore, the study on long-term memory from cognitive perspective is a bold step, and we think there are much room for further research in this field.

\section{Acknowledgements}

This work is supported by Xi'an Shiyou University Youth Science and Technology Innovation Fund (2013QN011).

\section{References}

[1] Watson, Nicholas (1999). Human Memory: Basic Concepts and Principles. New York: Warner Book.

[2] Carruthers, M. (1990). The Book of Memory. Cambridge: Cambridge University Press.

[3] Tulving, E. (1972). Organization of Memory. New York: Academic Press.

[4] Seamon, John G (1980) Memory and Cognition. New York: Oxford University Press.

[5] Setton, Robin (1999) Simultaneous Interpretation: A Cognitive-pragmatic Analysis. Amsterdam: John Benjamins Publishing Company.

[6] Miller, G. A (1956). The magical number seven, plus or minus two: Some limits of our capacity for processing information. Psychological Review, 63, 81-97.

[7] Barbara Moser- Mercer (1978). Language Interpretation and Communication. New York: Plenum Press.

[8] Beddeley, A. D (1990). Working Memory. Oxford: Oxford University Press.

[9] Anderson, J. R (1985) Learning and memory: An integrated approach. New York: John Wiley \& Sons. Hebb, D. O. (1961). Concerning imagery. PsychologicalReview.75, 466-477.

[10] Roderick, Jones (2008). Conference Interpreting Explained. Shanghai: Shanghai Foreign Language Education Press.
[11] Craik, F. LM. \& Lockhart, R. S. (1972) "Levels of Processing: A Framework for Memory Research", Journal of verbal Learning and verbal Behavior, 11, 671-684.

[12] Phelan, M. (2001). “the Interpreter's Resource”, Multilingual Matters LTD.

[13] Becker J D (1975). "In Proceedings of the Conference on Theoretical Issues in Natural Language Proceeding." The Phrasal Lexicon. 7: 70-77.

[14] Moser-Mercer, (1997). Process models in simultaneous interpretation. New York: Plenum Press.

[15] Tarpy, Roger (1978). Foundations of Learning and Memory. Illinois: Scott Fore manand Company.

[16] Stern, H. (1983). Fundamental Concepts of Language Teaching. Oxford: Oxford University Press.

[17] Gile, D. (1995). Concepts and Models for Interpreter and Translator Training. Amsterdam\& Philadelphia: John Benjamins.

[18] An Xinqui (2004). The Memory in Interpretation. Chinese Science \& Technology Translators Journal, 4, 22.

[19] Bao Gang (1998). Interpretation Studies. Beijing: Tourism Education Press.

[20] Baogang. (1999). Comprehension in Interpretation .Journal of Beijing International Studies University, 3, 1-12.

[21] Bao xiaoying (2005). The Application of Psychology in Interpretation Teaching. Foreign Language World, 3, 37-42.

[22] Gao Fen (2007). The Cognitive Analysis of Memory in Interpretation Teaching. Journal of Shaanxi Normal University, 2, 364-366.

[23] Gao Bin \& Chai Mingjiong (2009). New Development in the Study of Simultaneous Interpreting in the West---A Bibliometric Study. Chinese Translators Journal, 2, 17-21.

[24] Han Ling (2007). The Improvement of Memory and Interpretation Teaching. Journal of Fu Yang Teachers College, 6, 116-119.

[25] Han Xiaoming (2004). Memory Training in Interpretation Teaching Based on Memory Mechanism. Journal of Chongqing Institute of Technology, 6, 156-157.

[26] Li Fangqin (2004). On the Strategies to Improve Memory. Chinese Science \& Technology Translators Journal, 4, 17-20.

[27] Liu Heping (2011). Interpreting: Teaching Method of Thinking in Scientific Reasoning and Interpretation. Beijing: China Translation and Publishing Corporation.

[28] Liu Jinyu (2009), The Study of Memory Model in Interpreting and Cognitive Psychological Factors. Journal of Inner Mongolia Agricultural University. 2, 143-146.

[29] Ma Yingmai \& Sun Changyan (2004). Memory and Comprehension in Interpretation. Journal of Ningxia University, 4, 78-79.

[30] Tang Simin (2006). Memory Training in Interpretation. Journal of Jiaying University, 4, 119-122.

[31] Wu Yicheng (2001). A Review of Cognitive Processes in Translation and Interpreting. Modern Foreign Languages, 3, 324-330. 
[32] Xu Mingying (2006). The Improvement of Memory in Interpreting. Journal of Fujian Normal University, 4, 50-52.

[33] Xu Ming (2008). Overview of Interpreting Studies in the West. Chinese Translators Journal, 1, 16-21.

[34] Xu Ming (2010). Cognitive Processes in the "deverbalization" of the Cognitive Approach. Chinese Translators Journal, 3, 511 .
[35] Xiao Xiaoyan (2001). The Multi-Task Processing Model in Simultaneous Interpretation. Chinese Translators Journal, 2, 33-36.

[36] Zhang Wei (2006). Interpretation and Memory. Foreign Languages Research, 6, 66-70.

[37] Zhong Weihe. (2003). Knowledge Requirements for Interpreters and Their Implication to Interpreting Course Designing. Chinese Translators Journal, 4, 63-65. 